\title{
A Search for New Variable and Proper Motion Stars in the Galactic Disk
}

\author{
Robert A Benjamin ${ }^{1}$ \\ University of Wisconsin-Whitewater \\ Whitewater, WI
}

\begin{abstract}
Mid-infrared observations of sections of the Galactic Plane with the NASA Spitzer Space Telescope at two widely spaced epochs (2004-5) and (2012-13) have opened up the possibility of a large-scale search for variable stars, proper motion stars, and transients. This has required developing a new research infrastructure to merge and compare the two epochs of observations, and has led to the discovery of a surprising number of variable sources (over 100,000) and high proper motion sources (over 400). We quantify the probability that a star observed in the midinfrared will show variability at a certain amplitude. Stars with apparent magnitude fainter than $10^{\text {th }}$ magnitude (in the [3.6] micon bandpass) have a $0.6 \%$ probability of varying by more than 0.25 magnitudes. This probability increases to $14 \%$ for stars brighter than $6^{\text {th }}$ magnitude. We also describe upcoming observations of the center of the Milky Way Galaxy.
\end{abstract}

\section{The Time Variable Milky Way Galaxy}

One of the new frontiers of astrophysics is time-domain astronomy. Many stars flicker and occasionally flare, gamma ray and X-ray sources burst both within and outside the Galaxy, and supernovae explosions are now regularly recorded in distant galaxies across the universe. Even ordinary stars can change their brightness when eclipsed by companion stars or planets. Due to convection and sunspots, at the micro-magnitude level all stars are variable. The value of studying such sources has been demonstrated by NASA missions as varied as Kepler, which has statistically assessed the probability of stars having planets, to the Wide-field Infrared Sky Survey (WISE), an all-sky survey that has the potential to yield tens of thousands of new variable stars (Klein et al. 2011). Ground-based projects such as the LSST (Large Synoptic Survey Telescope), the top priority ground-based project in the 2010 astronomy decadal report, will survey the sky repeatedly. This mission will produce as much data every night as can be found in the entire Library of Congress, and will unveil the time-variable sky, with the only limitation being the significant dust-extinction in the Galactic plane.

Thanks to our work with the on-going GLIMPSE survey, a mid-infrared survey of the Galactic plane using NASA's Spitzer Space Telescope, we have amassed a database of over 239 million sources (Benjamin et al 2003; Churchwell et al 2009). The chief advantage of infrared wavelengths is that the effects of dust extinction are minimal on the observations. Stars that are completely invisible in the optical can often be very bright in the infrared. In the last few years,

\footnotetext{
${ }^{1}$ This work was supported by a 2014 Research Infrastructure Grant from the Wisconsin Space Grant Consortium. The author would like to acknowledge the invaluable help of Dr. Juliana Constantinescu (University of WisconsinWhitewater) for her help in creating class activities to allow her students to confirm our variable stars and proper motion candidates, Brian Babler (University of Wisconsin-Madison) for his work in providing the matched source lists and blinking images, and UW-Whitewater students Brandon Behnke, Kwun Ming Chan, and Fenglin Xi for their help in researching the proper motion stars and doing the optical follow-up work at WIYN $0.9 \mathrm{~m}$ for variable star candidates.
} 
we were fortunate enough to be able to re-survey parts of the Galactic disk first surveyed in 2004-2005. This resulted in over150 square degrees overlap with over 27.7 million sources in common. A comparison between the catalogs for these two surveys led to the identification of over 140,000 variable star candidates. This was a completely new undertaking for us, and required the development of a new research infrastructure.

\section{Finding and Confirming Variable Stars in the Galaxy}

The goal of the original GLIMPSE program (Benjamin et al. 2003) was three-fold: (1) a complete census of the (luminous) stellar content of the Galaxy, (2) mid-infrared coverage of the stellar, proto-stellar, and diffuse emission of a huge number of star forming regions in the Galactic plane, and (3) discovery of new classes of objects that had previously been hidden by the large columns of interstellar dust. On all three counts, this program was successful (Churchwell et al 2009). It confirmed the previously disregarded "Long Bar" of the Galaxy (Benjamin et al 2005), rekindled interest in spiral structure of the Galaxy (Benjamin 2008), allowed for estimates of the current star formation of the Galaxy based on young stellar objects (YSOs; Robitaille \& Whitney 2010), and has yielded large catalogs of new classes of objects including infrared dark clouds (Peretto \& Fuller 2009), stellar outflows from high mass protostars (Cyganowski et al 2008), and high mass YSOs and asymptotic giant branch (AGB) stars (Robitaille et al 2008). These catalogs provide uniform samples to study the large-scale star formation of the Milky Way in a statistical meaningful way.

Deep GLIMPSE re-surveyed the inner part of the Milky Way galaxy from March 2012 to March 2013, following the changing latitude of the warp on the far-side of the Galactic disk. The original GLIMPSE survey, which covered galactic longitudes $|1|<65^{\circ}$ and latitudes $|b|<1^{\circ}$ did not cover the warp extending up to $|\mathrm{b}|<3^{\circ}$. Deep GLIMPSE partially overlapped with this original survey; the overlap regions had 27.7 million sources in common.

More than 100,000 sources, $0.36 \%$ of the total, are variable at the 2 -sigma level for all measured bands (with a minimum of two bands). Figure 1 shows the probability that a source of a given magnitude will show variability at a given amplitude. Determining what type of sources these are has become one of our principal projects. Only 56\% had 2MASS K band (2.2 micron) measurements, and only $3 \%$ of these variables were matched to known objects within 10 arcseconds using the astronomical database SIMBAD. In the matched sample, our variable sources include 618 AGB stars, 182 sources in the vicinity of star-forming dark clouds, 128 YSOs, and 117 previously known variable stars. Over the summer of 2015, we selected a continous $1^{\circ} .5 \times 0^{\circ} .5$ region of the Galactic plane for nightly optical monitoring with the WIYN $0.9 \mathrm{~m}$ telescope to confirm the variability and obtain further constraints on a subset of these variable stars. The analysis of these data are ongoing. 


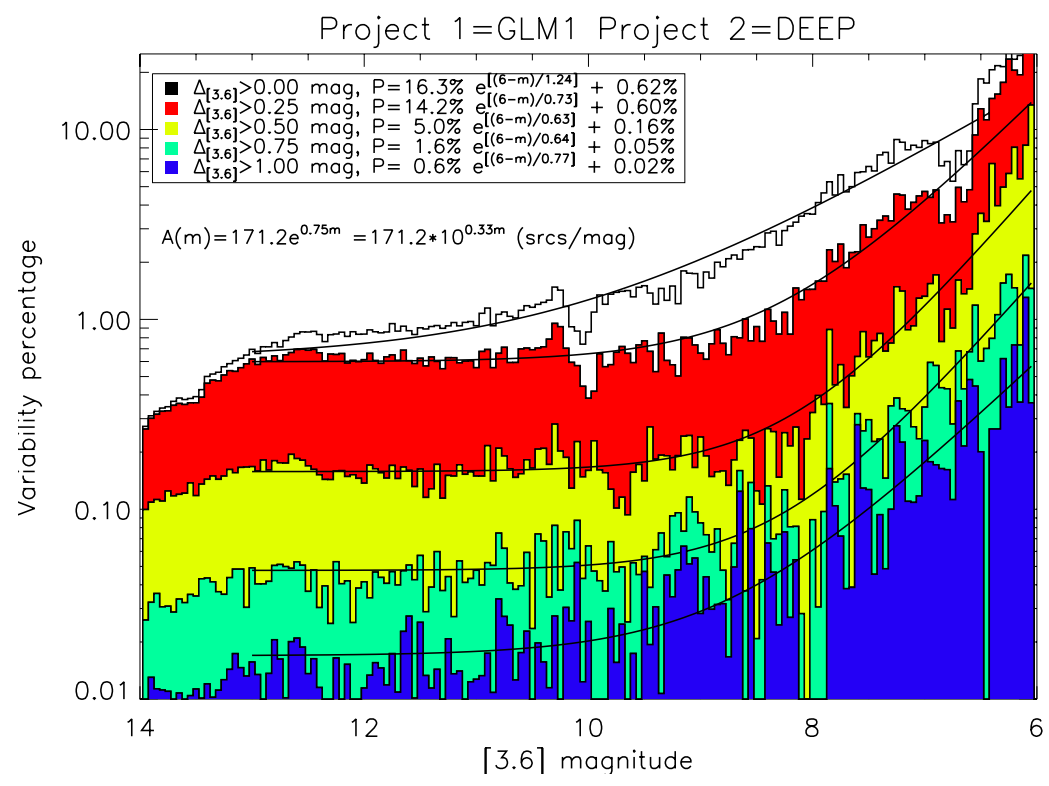

Figure 1. The probability of a star of a given observed [3.6] magnitude $(\mathrm{m})$ being variable as a function of observed magnitude for different variability amplitudes. For stars fainter than $\mathrm{m}_{=} 10$, the variability probability asymptotes to a fixed value that ranges from $0.02 \%$ (for variability amplitudes greater than $1.0 \mathrm{mag}$ ) to $0.6 \%$ (for variability amplitudes greater than $0.25 \mathrm{mag}$ ). For sources brighter than $\mathrm{m}=10$, the probability of a source being variable increases by a factor of 30 by the time one reaches $m=6$. Presumably a large fraction of these brighter sources are asymptotic red giant branch stars.

\section{A Surprising Number of Proper Motion Stars}

While confirming our variable stars via visual inspection, we began to notice many proper motions source, i.e. sources that moved noticeably with respect to background stars over a decade's time. Such sources are generally assumed to be nearby, and given the fact that many of them are bright in the infrared but not seen in the optical wavelengths, they are probably lowmass stars or substellar "brown dwarfs" (or else very distant, high- or hyper-velocity stars). There are many reasons these objects are of potential scientific importance so we redirected our program to focus on these objects. Figure 2 shows the motion of one newly discovered source on the sky and how well our measurements of proper motions compare to previous works.

We found 395 high proper motion stars $>100$ milli-arcseconds/year down to a limiting infrared magnitude of $m_{[3.6]}=13$ of which nearly three-quarters are new discoveries. Spectral follow-up of three of these sources (so far) have uncovered three mid-to-late $\mathrm{M}$ dwarfs, one of which (GLIMPSE 1713-3952) is known to have X-ray flares and is likely to be only $20 \mathrm{pc}$ from the Sun. Of our new detections, all but five could be associated with a source in the 2MASS catalog that confirmed the proper motion at a third epoch in time. All of these objects are in areas of the sky that had been optically surveyed for proper motion in the past without detecting these sources, indicating that mid-infrared observations are sensitive to sources that escape detection in other wavelengths. 

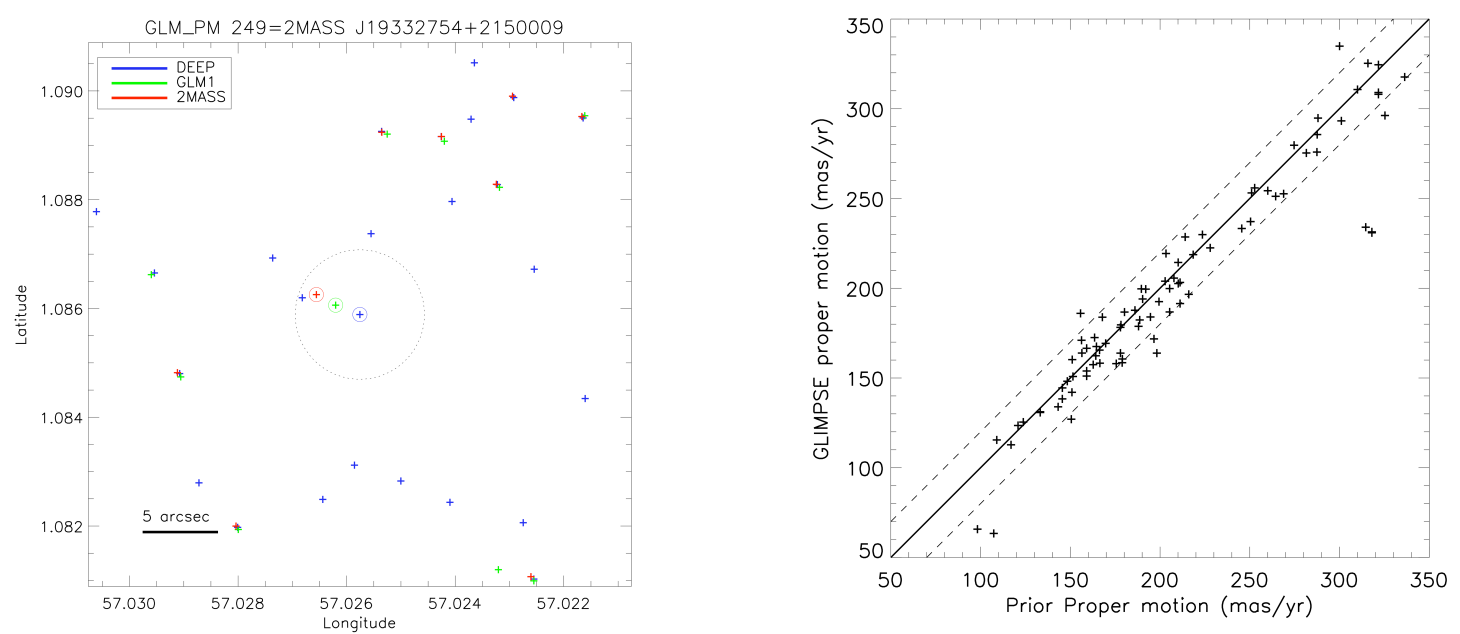

Figure 2 [Left]. The proper motion of GLIMPSE 1933+2150, a new late M/early L companion to the G2V star HD 184385 located $25 \mathrm{pc}$ from the Sun. [Right] A comparison of GLIMPSE proper motions to published proper motions for recovered sources. The diagonal band shows that most sources agree to within 20 mas/year.

\section{One Final GLIMPSE}

The discovery of so many high proper motion objects made us aware of the scientific value of obtaining a second epoch of observations to the Galactic center direction with the Spitzer Space Telescope. Thanks to the research program described in this work we were able to successfully propose for 97 additional hours on Spitzer to re-image the most extincted, most crowded section of the Milky Way (Figure 3,4), where no other telescopes have done systematic proper motion studies. This project has several scientific goals:

(1) Measure the stellar velocity dispersion both parallel and perpendicular to the Galactic plane as a function of (1) direction and (2) depth. By defining samples of red clump giants or red giant branch stars of different magnitudes, we will probe different depths through the Galactic bulge (Nidever et al 2012).

(2) Search for streaming motions in the mean proper motion of sources as a function of Galactic latitude and longitude. The central bar of the Milky Way is complex, consisting of central "triaxial bulge" , an "X-shaped" vertical structure, and a thin in-plane "Long Bar" (confirmed with GLIMPSE data in Benjamin et al. 2005). The dynamical models that produce these structures predict different proper motions for front-side and back-side sections. These have been potentially observed (again, well off the plane) by Sumi et al (2013) and Poleski et al (2013).

(3) Search for brown dwarfs in crowded fields. Brown dwarfs, being nearby, have high proper motions and relatively isotropic distribution on the sky. Because of stellar crowding, the Galactic plane is the hardest place in the sky to look for such objects. As a result, there may still be interesting objects there that can not be detected with WISE because of the severe confusion WISE would face in the inner Galaxy. Our experience with detecting proper motion sources further out in the disk suggest we will find about 150 to 250 of these objects in the proposed survey. We have developed a collaboration with Dr. Davy Kirkpatrick to obtain spectroscopic follow-up of interesting sources. 


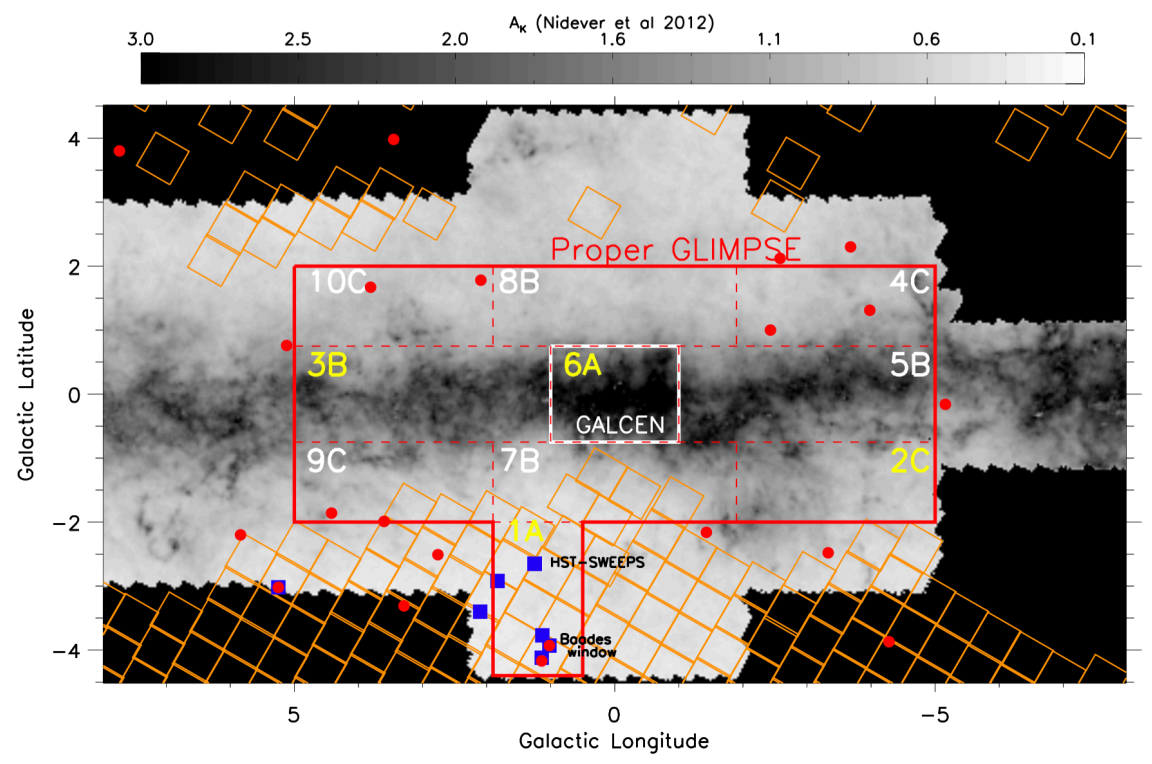

Figure 3. A view of our approved proper motion survey area superimposed on a two-dimensional dust extinction map generated using GLIMPSE data (Nidever et al 2012). Small optical windows observed in previous proper motion studies are shown with blue squares. Large-area coverage by the optical survey OGLE III (Udalsky et al 2008) is shown with orange tiles, and the location of globular clusters are noted with red circles. At mid-infrared wavelengths, the vast majority of this area has less than one magnitude of extinction. The central (GALCEN) region and Baade's window (Priority A) require 18.0 of observing time. The fields adjacent to GALCEN require 45 hours. The diagonal corners of our survey area will require 34.5 hours.

(4) Search for future microlensing events. One of the holy grails of brown dwarf science is obtaining empirical mass measurements against which to compare to theory. It has often been stated that such mass measurements are possible only in binary systems whose orbital parameters are well constrained. However, the discovery of brown dwarfs against the Galactic Plane makes it possible, in special cases, to measure the masses of single brown dwarfs. For brown dwarfs whose astrometric paths can be measured with milliarcsecond accuracy, candidate lensing events of background stars by the brown dwarf can be predicted in advance (e.g., Lepine \& DiStefano 2012).

(5) Search for hyper-velocity stars. An ever-growing sample of "hyper-velocity" stars have been found in the Galactic halo (Brown et al 2012). These stars have speeds up to $800 \mathrm{~km} / \mathrm{s}$ and are generally assumed to be ejected when a binary system near the supermassive black hole of the Milky Way is disrupted (Rossi et al 2014). There is some support for this idea given the presence of large number of young stars near Galactic center (Fritz et al 2014). Since we will be carrying out a uniform and symmetric proper motion survey of the bulge which is practically unaffected by extinction, we expect to be able to put meaningful limits on these models. The most exciting possibility is that we will catch one of these stars close enough to the Galactic Center to place constraints on this ejection scenario.

Decades-long proper motion surveys are rarely planned in advance, but the longevity of the Spitzer Space Telescope, the IRAC camera, and the GLIMPSE pipeline allow for the possibility of making nearly identical observations and data products a decade later, a rarity in Galactic 
astronomy. This was not an avenue we anticipated, but the WSGC Research Infrastructure program has allowed us to go down an interesting new road in Galactic astronomy.

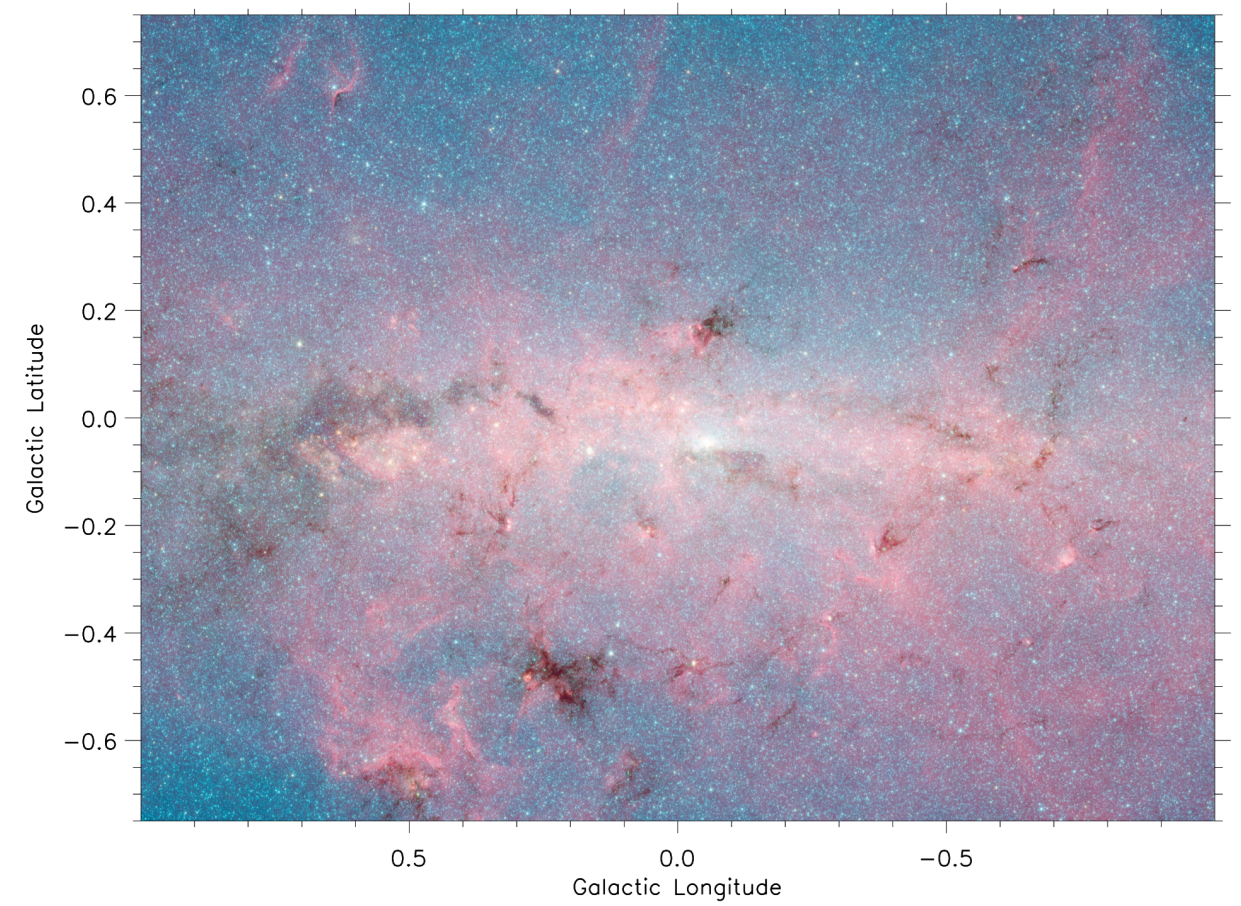

Figure 4. Mid-infrared image of the GALCEN area $\left(|1|<1^{\circ}\right.$ and $\left.|\mathrm{b}|<0 .{ }^{\circ} 75\right)$ (PI: S. Stolovy). This area will be observed with same procedure as in 2005: five-dither, two-second frame time observations. The observing plans we have provided are comparable to the original GALCEN observations. Since this area was never re-observed by Spitzer, we will also be providing new constraints on mid-infrared stellar variability in this region.

\section{References}

Benjamin, R.A. et al 2003, Publications of the Astronomical Society of the Pacific 115, 953.

Benjamin, R.A. et al 2005, Astrophysical Journal Letters, 630, 149.

Benjamin, R.A. 2008, in Astrophysical Society of the Pacific Conference Volume 387, 375.

Brown, W.R. et al 2012, Astrophysical Journal Letters, 754, 2.

Churchwell, E.B. et al 2009, Publications of the Astronomical Society of the Pacific, 121, 213.

Cyganowski, C. J. et al 2008, Astronomical Journal, 136, 2391.

Fritz, T.K. et al 2014, arXiv.org/1406.7568.

Klein, C. R., Richards, J. W. Butler, N. R., Bloom, J. S. 2011, Astrophysical Journal 738, 185.

Lepine, S. \& Di Stefano, R. 2012, Astrophysical Journal, 749, 6.

Nidever, D. et al 2012, Astrophysical Journal Supplement, 201, 35.

Peretto, N. \& Fuller, G.A. 2009, Astronomy \& Astrophysics, 505, 405.

Poleski, R. et al 2012, Astrophysical Journal, 776, 76.

Robitaille, T.P. et al 2008, Astronomical Journal, 136, 2413.

Robitaille, T.P. \& Whitney, B.A. 2010, Astrophysical Journal Letters, 710, L11.

Rossi, E.M. et al 2014, Astrophysical Journal, 795, 125.

Sumi, T. et al 2013, Monthly Notices of the Royal Astronomical Society, 432, 124. 